\title{
Barbed sutures versus conventional tenorrhaphy in flexor tendon repair: An ex vivo biomechanical analysis
}

\author{
Özlem Çolak ${ }^{1}$, Yüksel Kankaya ${ }^{2}$, Nezih Sungur², Kadri Özer ${ }^{3}$, Koray Gürsoy ${ }^{2}$, Kemal Şerbetçi ${ }^{4}$, \\ Uğur Koçer ${ }^{2}$ \\ ${ }^{I}$ Department of Plastic, Reconstructive and Aesthetic Surgery, Okmeydani Training and Research Hospital, İstanbul; ${ }^{2}$ Department of Plastic, \\ Reconstructive and Aesthetic Surgery, Ankara Training and Research Hospital, Ankara; ${ }^{3}$ Department of Plastic, Reconstructive and Aesthetic \\ Surgery, Aydin State Hospital, Aydın; ${ }^{4}$ Department of Biomedical Engineering, İzmir Democracy University, İzmir, Turkey
}

Background The management of flexor tendon injuries has evolved in recent years through industrial improvements in suture materials, refinements of repair methods, and early rehabilitation protocols. However, there is no consensus on the ideal suture material and technique. This study was conducted to compare the tensile strength, repair time, and characteristics of 4-strand cruciate, modified Kessler, and 4-strand horizontal intrafiber barbed sutures for flexor tenorrhaphy with a 12-mm suture purchase length in an animal model.

Methods The right third deep flexors of 60 adult Leghorn chicken feet were isolated and repaired with a 12-mm suture purchase length. The tendons were randomly assigned to three groups of equal number ( $n=20$ each). Groups 1 and 2 received 4-strand cruciate and modified Kessler repair with conventional suture materials, respectively. A 4-strand horizontal intrafiber barbed suture technique was used in group 3. The repaired tendons were biomechanically tested for tensile strength, 2-mm gap resistance, and mode of failure. Repair times were also recorded.

Results The maximum tensile strength until failure was $44.6 \pm 4.3 \mathrm{~N}$ in group $1,35.7 \pm 5.2 \mathrm{~N}$ in group 2, and $56.7 \pm 17.3 \mathrm{~N}$ in group 3. The barbed sutures were superior to the other sutures in terms of the load needed for $2-\mathrm{mm}$ gap formation $(\mathrm{P}<0.05)$. Furthermore, the barbed sutures showed the shortest repair time $(P<0.05)$.

Conclusions This study found that 4-strand horizontal intrafiber barbed suture repair with a 12-mm purchase length in a chicken flexor tendon injury model showed promising biomechanical properties and took less time to perform than other options.

Keywords Suture techniques / Instrumentation / Tendon injuries / Surgery
Correspondence: Özlem Çolak Department of Plastic, Reconstructive and Aesthetic Surgery, Okmeydani Training and Research Hospital, Darülaceze Cad. No:25 Okmeydani, İstanbul 34384, Turkey Tel: +90-212-314-5555 Fax: +90-212-221-7800 E-mail:drozlemcolak@hotmail.com

Received: 10 Aug 2018 • Revised: 16 Jan 2019 • Accepted: 16 Feb 2019

pISSN: 2234-6163 • elSSN: 2234-6171 • https://doi.org/10.5999/aps.2018.00962 • Arch Plast Surg 2019;46:228-234

\section{INTRODUCTION}

The management of flexor tendon injuries has evolved in recent years through industrial improvements in suture materials, refinements of repair methods, and early rehabilitation protocols [1-6]. However, there is no consensus on the ideal suture mate- 
rial and technique, which would provide low friction with minimal bulk and sufficient tensile strength to allow early motion without any risk of failure $[3,7]$. Currently, the most popular method of zone II flexor tendon repair is a minimum of 4-strand conventional knotted core repair with braided, nonabsorbable sutures $[7,8]$. The major concerns about conventional knotted repair are knot size and bulk at the repair site, which prevent tendon gliding and early motion. Moreover, inadequate suturetendon interactions at the loop sites and suture knot failure could limit the tensile strength at the repair site [1].

Advances in suture materials reintroduced the possibility of using barbed sutures for tendon repairs. The advantages of barbed tenorrhaphy are minimal bulk and lower gliding resistance due to the knotless design, better load distribution along the entire suture length caused by the barbs locking into the tendon, and improved tendon flow resulting from the reduction of constricting forces $[1,3,5]$. Furthermore, the barbed suture technique is quicker than other techniques, and is easy to perform and less demanding in terms of surgical skills.

Several factors contributing to the initial strength of flexor tendon repairs have been identified. These mainly include material properties, knot security, suture components, the number of strands, and the length of suture purchase $[6,9,10]$. The core suture component yields primary resistance to gap formation and failure at the repair site. Recent biomechanical studies demonstrated that both the number of strands and the length of core suture purchase were correlated with the baseline and ultimate tensile strength of repairs $[5,7,9,11]$.

Barbed and traditional suture materials have been compared, and conflicting results have been reported in previous studies with variable length of suture purchase $[2,6,8,12-14]$. The use of barbed sutures as a tenorrhaphy material has been increasingly accepted, since they theoretically allow smoother gliding under pulleys as a result of the lower cross-sectional area under load, the improved load distribution along the entire length of the suture, and the better tendon flow caused by the reduction of constricting forces $[1,15]$. In clinical practice, however, the cruciate-locked and modified Kessler techniques with nonabsorbable suture materials are still the most frequently used techniques. In this paper, we compared the tensile strength, repair time and characteristics of the two most utilized conventional tenorrhaphy methods with an alternative suturing method using the horizontal intrafiber barbed suture technique for flexor tenorrhaphy in an animal model to provide a more comprehensive overview of the effectiveness of barbed sutures in flexor tendon repair compared with conventional methods. Although a few studies have compared barbed sutures to conventional suture tenorrhaphy, no study has compared these three groups, which is a unique aspect of our study compared to other biomechanical studies. The purpose of this study was to compare the tensile strength, repair time, and characteristics of 4-strand cruciate, modified Kessler, and 4-strand horizontal intrafiber barbed suture repair for flexor tenorrhaphy with a 12-mm suture purchase length in an animal model.

\section{METHODS}

\section{Tendon harvest and preparation}

Adult white Leghorn chickens weighing between 2 and $4 \mathrm{~kg}$ were used in this experimental study, since their flexor mechanism is similar to that of human digits. All procedures were conducted under protocols approved by the Ankara Research and Training Hospital's Committee on the Use of Animal Subjects in Research in accordance with the National Institutes of Health Guide for the Care and Use of Laboratory Animals (IRB No. 0509/4253). A midline longitudinal incision was made over the volar surface of the right third digit. The flexor sheath was dissected along the pulleys and deep flexors of 60 chicken feet were isolated (Fig. 1). Standardizing the length to be $6 \mathrm{~cm}$, the tendons were randomly assigned into three groups of equal number $(n=20$ each). Group 1 underwent the 4-strand cruciate technique (Fig. 2A), group 2 received modified Kessler repair (Fig. 2B), and the horizontal intrafiber barbed suture technique (Fig. 2C) was used in group 3. After the ends of the tendons were fixed to the table, the middle point was marked and a full cut was made from that point (Fig. 3).

\section{Tendon repair}

Repairs with a 12-mm suture purchase length were performed by a single surgeon immediately after the incision was made.

\section{Fig. 1. Isolation of the deep flexor tendons}

$(A, B)$ The deep flexor tendons of 60 chicken feet were isolated. (C, D) The flexor sheath was dissected along the pulleys.
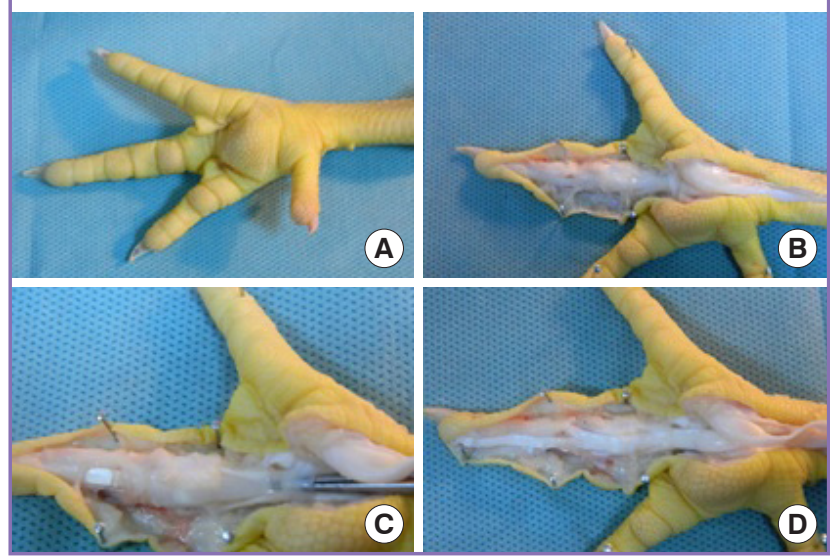
Groups 1 and 2 received 4-strand cruciate and modified Kessler repair, respectively, as described before (Fig. 4 A and B) $[7,12$, 14]. In group 3 , the needle was introduced into one side of the repair $12 \mathrm{~mm}$ away from the cut point and passed through the tendon, parallel to the direction of the fibrils. After traversing the injury site, it was advanced for $12 \mathrm{~mm}$ before exiting the tendon surface on the other side. The needle was then reintroduced into the end of that tendon, providing a transverse pass in retrograde fashion that yielded two-strand horizontal repair. Next, starting from the other side, the horizontal intrafiber repair procedure was repeated, so that 4-strand repair was achieved (Fig. 4C). For the tendons in the first two groups, 3-0 polypropylene suture material (Prolene; Ethicon Inc., Somerville, NJ, USA) with $3 / 8$ circle cutting needles was used, and in the third group, 3-0 unidirectional barbed suture material with $3 / 8$ circle cutting needles (V-Loc; Covidien Deutschland GmbH, Neustadt, Germany) was used. For all repairs, the repair time, which was defined as the time from the tendon cut to the end of tenorrhaphy, was recorded.

\section{Biomechanical assessment}

In order to standardize the experimental conditions, all repaired tendons were preserved at room temperature prior to the mechanical tests. The repaired tendons were tested for tensile strength on the Lloyd LRX5K Mechanical Tester (Lloyd Instruments, Fareham, UK) with grasping clamps. During the test, tendons were sprayed with physiological saline solution to prevent dryness and to keep their mechanical properties unchanged. Pretesting with loads up to $500 \mathrm{~N}$ was performed in order to ensure that there was no tendon slippage. The tendons were preloaded to $2 \mathrm{~N}$ and tested under linear distraction at an average rate of $50 \mathrm{~mm} / \mathrm{min}$ to simulate the forces applied at the repair site during early active motion protocols $[2,8,12]$. Dis-

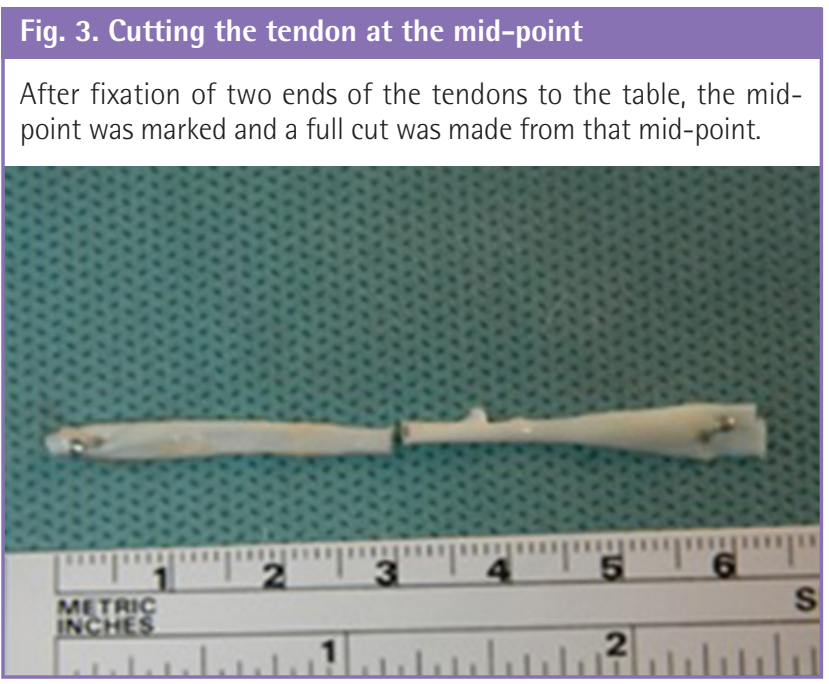

Fig. 2. The techniques used in the study

(A) The 4-strand cruciate technique was applied in group 1, whereas (B) group 2 received modified Kessler repair, and (C) the horizontal intrafiber barbed suture technique was used in group 3.
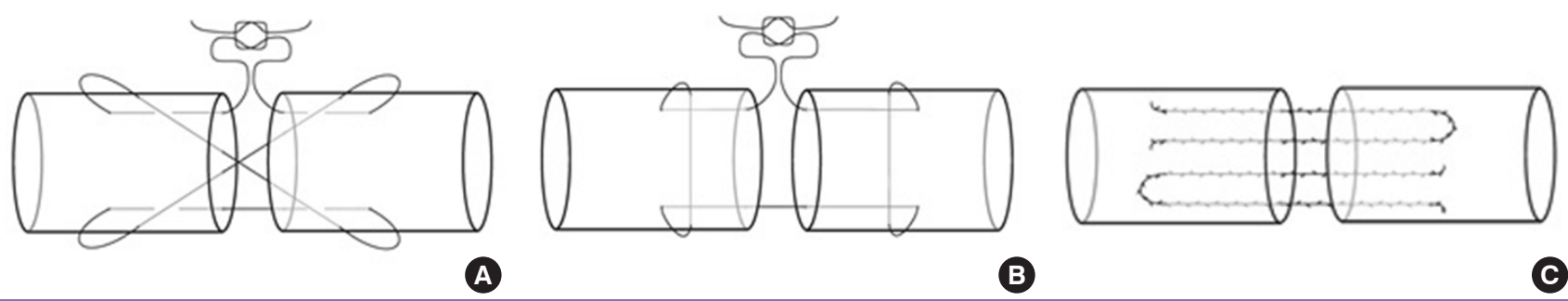

B

Fig. 4. Views of the tendons after repair

(A) The 4-strand cruciate technique was applied in group 1, whereas (B) group 2 received modified Kessler repair, and (C) the horizontal intrafiber barbed suture technique was used in group 3.
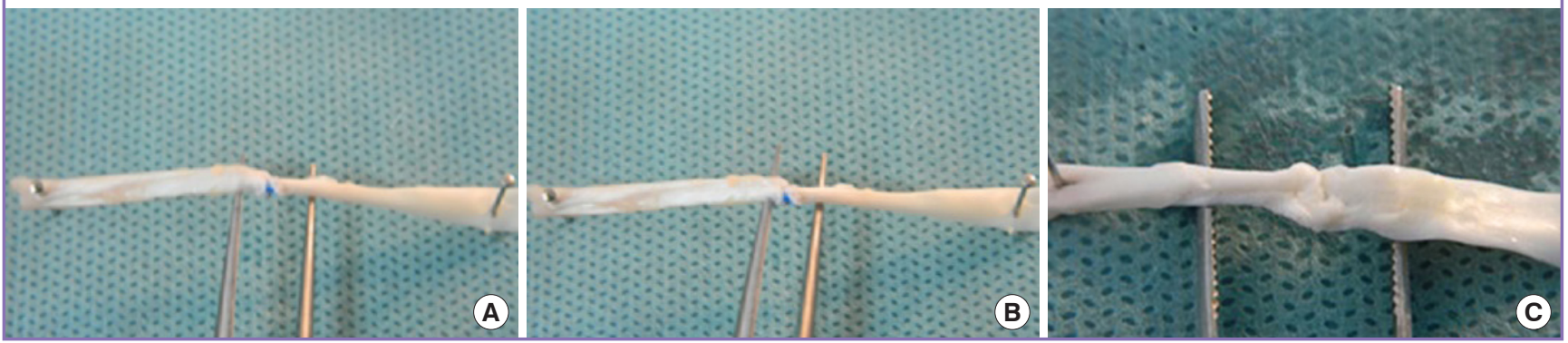
traction continued until mechanical failure of the repair site, which was defined as a sudden decrease in tensile strength or a detectable gap $>2 \mathrm{~mm}$. Frame-by-frame video records were synchronized with the applied force and displacement data. The load to failure and mode of failure (categorized as pullout, suture breakage, and knot rupture) were recorded (Fig. 5).

\section{Statistical analysis}

To compare group means and to evaluate significance among groups for 2-mm gap resistance, one-way analysis of variance was used. P-values $<0.05$ were considered to indicate statistical significance. Further analysis of groups was performed using the post hoc Turkeys test.

\section{RESULTS}

\section{Repair time}

The 4-strand cruciate repair group (group 1) showed significantly longer repair times than the other groups, with an average of $294.5 \pm 7$ seconds. The average repair time was $240.7 \pm 6.6$ seconds in the modified Kessler repair group (group 2). The horizontal intrafiber barbed suture group (group 3 ) showed the fastest repair time, with an average of $195.7 \pm 9$ seconds. This difference was statistically significant $(\mathrm{P}<0.05)$ (Fig. 6).

\section{Fig. 5. Biomechanical assessment of repaired tendons}

(A) The tendons were preloaded to $2 \mathrm{~N}$ and tested under linear distraction at an average rate of $50 \mathrm{~mm} / \mathrm{min}$ to simulate the forces applied at the repair site. The video records were synchronized with the applied force and displacement data. (B) Load to failure and the mode of failure were recorded.
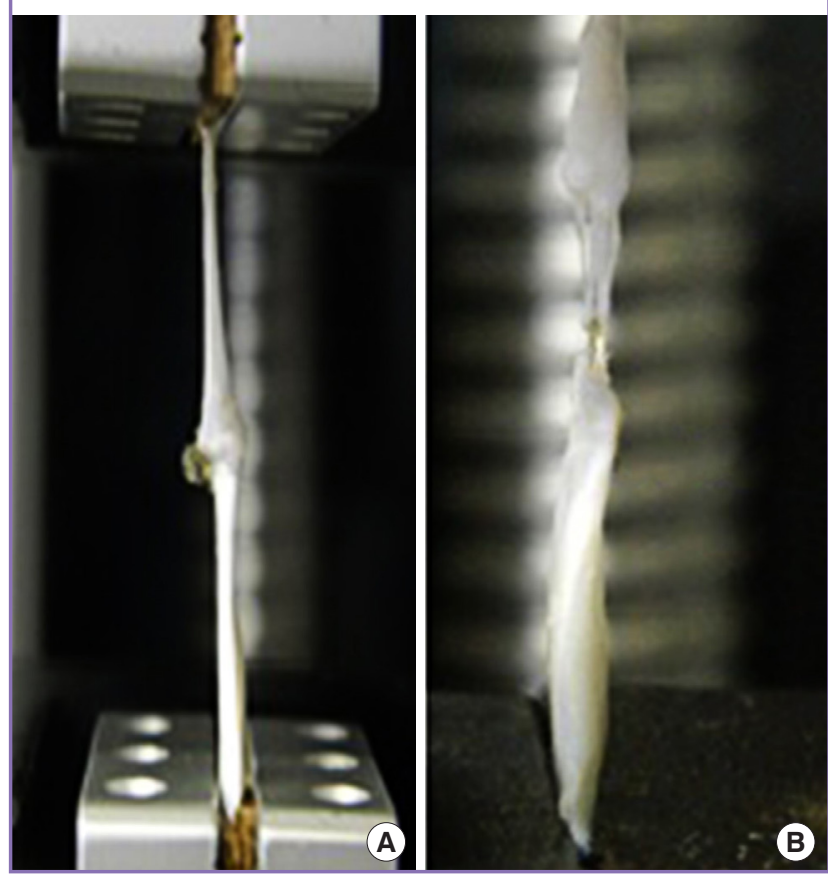

\section{Two-millimeter gap resistance}

The 2-mm gap resistance forces were measured to be $10.91 \pm$ $4.09 \mathrm{~N}$ in group $1,9.63 \pm 2.78 \mathrm{~N}$ in group 2 , and $15.68 \pm 4.78 \mathrm{~N}$ in group 3. Group 3 (4-strand horizontal intrafiber barbed sutures) was superior to the other groups in terms of the loads needed for 2-mm gap formation $(\mathrm{P}<0.05)$ (Fig. 7).

\section{Load to failure}

The maximum tensile strength until failure was $44.6 \pm 4.3 \mathrm{~N}$ in group 1, 35.7 $\pm 5.2 \mathrm{~N}$ in group 2, and $56.7 \pm 17.3 \mathrm{~N}$ in group 3 . Horizontal intrafiber barbed suture repair (group 3) yielded a higher ultimate tensile strength than was observed in the other groups $(\mathrm{P}<0.05)$ (Fig. 8, Supplemental Video 1).

\section{Fig. 6. Comparison of the average repair time}

The 4-strand cruciate repair group (group 1) showed a significantly longer repair time than the other groups, with an average of $294.5 \pm 7$ seconds. The horizontal intrafiber barbed suture group (group 3) showed significantly faster repair times than the other groups, with an average of $195.7 \pm 9$ seconds $(P<0.05)$.

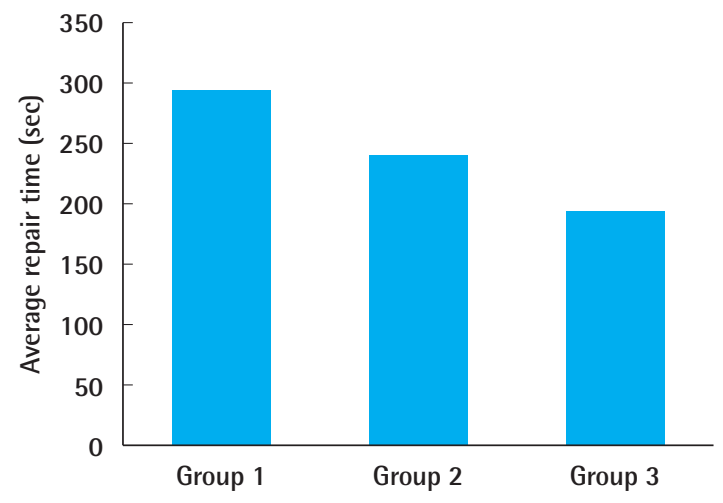

Fig. 7. Comparison of 2-mm gap resistance forces

The horizontal intrafiber barbed suture group was superior to other groups in terms of the loads needed for 2-mm gap formation $(\mathrm{P}<0.05)$

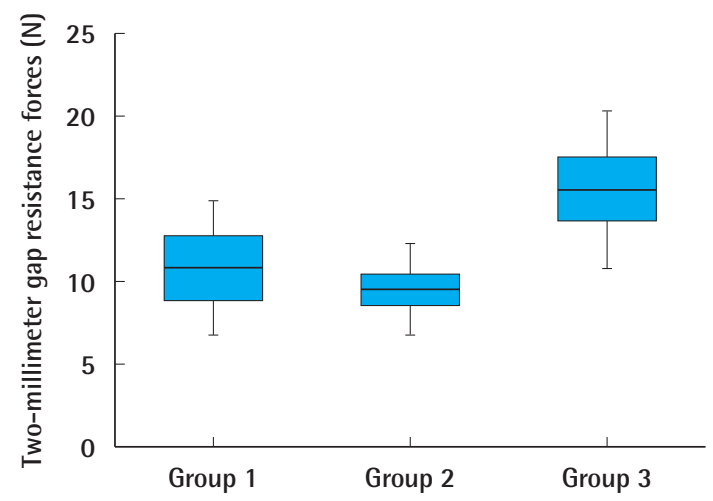




\section{Fig. 8. Comparison of maximum tensile strengths until failure}

Horizontal intrafiber barbed suture repair yielded a higher ultimate tensile strength than the other techniques $(P<0.05)$.

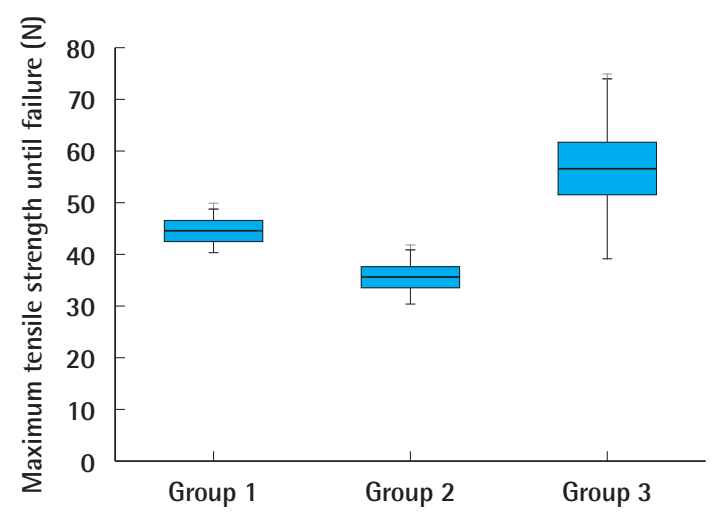

\section{Mode of failure}

The most common mode of failure was suture pull-out in all samples (90\%). Two cases of suture breakage were seen in each of the three groups (10\%). Suture knot unraveling was not seen in any of the repairs.

\section{DISCUSSION}

The prognosis for restoration of good function after flexor tendon repair is influenced mainly by repair strength and the postoperative forces experienced by the repair site. The initial strength of the repair depends on both the suture configuration and the type of suture material that is used as the construct. The optimum flexor tendon repair should be easy to perform and reliable, achieve strong enough tensile strength to allow early active motion, and have low gliding resistance $[2,6,14]$. Barbed suture models were intended for lifting and shaping of soft tissues. Fortunately, advancements in technology improved the utility of barbed sutures as knot-free devices for tendon repair. Barbed sutures theoretically offer safe and smooth passage along the direction of the barbs, whereas they possess strong resistance to motion against the direction of the barbs [1]. Nonetheless, their knotless design avoids creating weak points at locking zones, decreases the bulkiness at the repair site, and enables better distribution of the load along the entire length of suture purchase through the close barb-tendon interactions $[1,16]$. Through these mechanisms, tendon slippage at the repair site is eliminated as a source of failure.

Flexor tenorrhaphy with barbed devices was first reported in 1952 by Jennings and Bunnell, and the first scientific publication regarding the application of the barbed concept was conducted by McKenzie in 1967; however, these attempts did not gain popularity until the U.S. Food and Drug Administration approved barbed nylon, polydioxanone, and polypropylene sutures $[14,16]$. Advances in the commercialization of barbed products elicited great interest in its utility for various surgical procedures in aesthetical, abdominal, and gynecological surgery, despite cost considerations.

This ex vivo study compared the biomechanical properties and repair time of 4-strand horizontal intrafiber barbed suture repair with the well-known 4-strand modified Kessler and cruciate repair techniques with a $12-\mathrm{mm}$ suture purchase length in a chicken flexor tendon injury model. The repair time was significantly shorter in the barbed suture group than in the other groups, and the barbed suture technique yielded higher ultimate tensile strength than the others. Furthermore, in barbed suture repair, higher loads were needed for 2-mm gap formation.

Suture purchase length has been defined as the exit or entry distance of the suture from the cut ends of the tendon [17]. Only a few studies have investigated the effect of purchase length on the strength of flexor tendon repair. Merrell et al. [18] showed that the strength of the peripheral suture increased proportionally with increased length of purchase. In a biomechanical study of 74 fresh pig flexor tendons, Tang et al. [17] found the optimal length of purchase to be between 0.7 and $1.0 \mathrm{~cm}$ and that increasing the length of purchase from 0.7 to $1.2 \mathrm{~cm}$ did not increase the strength of the repair. However, none of those studies used barbed suture material for testing. In our study, the use of 4-strand horizontal intrafiber barbed sutures with a 12-mm purchase length might have contributed to the higher tensile strength of barbed sutures than other traditional repair techniques.

Regardless of the suture material and technique, the most common mode of failure in our study was suture pull-out. Previous studies have demonstrated that traditional repairs failed mostly by suture rupture, whereas barbed suture repairs most commonly failed by either suture pull-out or breakage $[3,7,11$, $14,16]$. The discrepancy in the mode of failure between our study and previous reports may be due to differences in the tendon model that was used and the suture purchase length for repairs. We used a chicken model because it has anatomical and biomechanical similarities to humans, in addition to its lower costs, and also because it involves fewer ethical difficulties than human cadaver models [19]. However, chicken flexor tendons are smaller and more difficult to manipulate for repair.

The barbed suture technique is quicker than other techniques, and is easy to perform and less demanding in terms of surgical skills. In cases of multiple tendon lacerations with other associated injuries, repair time is a matter of concern. Safe and quicker repairs can decrease the time in operating room, which is help- 
ful for both the surgeon and the patient, and can reduce the overall cost.

Our biomechanical study had some limitations. Due to ethical concerns in the current domestic environment, we were not able to perform this study with flexor tendons of human cadavers. Secondly, since the experiment was performed under $e x$ vivo conditions, it did not account for how the biomechanical properties of tendons could be affected by factors such as tissue ischemia, edema, and adhesion formation during the healing process. In addition, the lack of cyclic loading through a simulated active range of motion may have masked potential problems that could be encountered during postoperative rehabilitation in patients with flexor tendon injuries.

In conclusion, this study found that 4-strand horizontal intrafiber barbed suture repair with a $12-\mathrm{mm}$ purchase length in chicken flexor tendon injury model showed promising biomechanical properties and took less time to perform than other methods. Further in vivo studies with long-term follow-up are needed to clarify the effectiveness of barbed materials for flexor tendon injuries in common clinical settings.

\section{NOTES}

\section{Conflict of interest}

No potential conflict of interest relevant to this article was reported.

\section{Ethical approval}

All procedures were conducted under protocols approved by the Ankara Research and Training Hospital's Committee on the Use of Animal Subjects in Research in accordance with the National Institutes of Health Guide for the Care and Use of Laboratory Animals (IRB No. 0509/4253).

\section{Author contribution}

Study concept and design: all authors. Data acquisition: Çolak Ö, Özer K, Şerbetçi K. Data analysis and interpretation: Çolak Ö, Özer K, Şerbetçi K. Drafting of the manuscript: Çolak Ö, Özer K, Şerbetçi K. Critical revision of the manuscript for important intellectual content: Çolak Ö, Özer K. Statistical analysis: Çolak Ö, Özer K. Administrative, technical, or material support: Kankaya Y, Sungur N, Koçer U, Gürsoy K. Study supervision. Kankaya Y, Sungur N, Koçer U, Gürsoy K. Approval of final manuscript: all authors.

\section{ORCID}

Özlem Çolak https://orcid.org/0000-0002-0201-1649

Yüksel Kankaya http://orcid.org/0000-0003-1761-3324
Nezih Sungur https://orcid.org/0000-0002-9744-0007

Kadri Özer https://orcid.org/0000-0003-2966-6618

Koray Gürsoy https://orcid.org/0000-0002-7730-7225

Kemal Şerbetçi https://orcid.org/0000-0002-6646-5252

Uğur Koçer https://orcid.org/0000-0001-9873-1183

\section{Supplementary material}

Supplemental Video 1. Video showing failure in response to overloading of the breakage force in the barbed tendon group.

Supplemental data can be found at:

https://doi.org/10.5999/aps.2018.00962.v001

\section{REFERENCES}

1. Parikh PM, Davison SP, Higgins JP. Barbed suture tenorrhaphy: an ex vivo biomechanical analysis. Plast Reconstr Surg 2009;124:1551-8.

2. Sull A, Inceoglu S, August A, et al. Comparison of barbed sutures in porcine flexor tenorrhaphy. Hand 2016;11:475-8.

3. Engel JL, Gabra JN, Esterle AR, et al. In vitro comparison of two barbed suture configurations for flexor tendon repair. J Hand Microsurg 2017;9:126-30.

4. Cortez R, Lazcano E, Miller T, et al. Barbed sutures and wound complications in plastic surgery: an analysis of outcomes. Aesthet Surg J 2015;35:178-88.

5. Shin JY, Kim JS, Roh SG, et al. Biomechanical analysis of barbed suture in flexor tendon repair versus conventional method: systematic review and meta-analysis. Plast Reconstr Surg 2016;138:666e-674e.

6. Viinikainen A, Goransson H, Ryhanen J. Primary flexor tendon repair techniques. Scand J Surg 2008;97:333-40.

7. Chauhan A, Schimoler P, Miller MC, et al. Comparing biomechanical properties, repair times, and value of common core flexor tendon repairs. Hand 2018;13:313-8.

8. Nayak AN, Nguyen DV, Brabender RC, et al. A mechanical evaluation of zone II flexor tendon repair using a knotless barbed suture versus a traditional braided suture. J Hand Surg Am 2015;40:1355-62.

9. Cao Y, Zhu B, Xie RG, et al. Influence of core suture purchase length on strength of four-strand tendon repairs. J Hand Surg Am 2006;31:107-12.

10. Tang JB, Wang B, Chen F, et al. Biomechanical evaluation of flexor tendon repair techniques. Clin Orthop Relat Res 2001 ;(386):252-9.

11. Marrero-Amadeo IC, Chauhan A, Warden SJ, et al. Flexor tendon repair with a knotless barbed suture: a comparative biomechanical study. J Hand Surg Am 2011;36:1204-8.

12. Clemente A, Bergamin F, Surace C, et al. Barbed suture vs 
conventional tenorrhaphy: biomechanical analysis in an animal model. J Orthop Traumatol 2015;16:251-7.

13. O'Brien FP 3rd, Parks BG, Tsai MA, et al. A knotless bidirectional-barbed tendon repair is inferior to conventional 4-strand repairs in cyclic loading.J Hand Surg Eur Vol 2016; 41:809-14.

14. Peltz TS, Haddad R, Scougall PJ, et al. Performance of a knotless four-strand flexor tendon repair with a unidirectional barbed suture device: a dynamic ex vivo comparison. J Hand Surg Eur Vol 2014;39:30-9.

15. Joyce CW, Whately KE, Chan JC, et al. Flexor tendon repair: a comparative study between a knotless barbed suture repair and a traditional four-strand monofilament suture repair.J Hand Surg Eur Vol 2014;39:40-5.
16. Joyce CW, Sugrue C, Chan JC, et al. A barbed suture repair for flexor tendons: a novel technique with no exposed barbs. Plast Reconstr Surg Glob Open 2014;2:e237.

17. Tang JB, Zhang Y, Cao Y, et al. Core suture purchase affects strength of tendon repairs. J Hand Surg Am 2005;30:12626.

18. Merrell GA, Wolfe SW, Kacena WJ, et al. The effect of increased peripheral suture purchase on the strength of flexor tendon repairs. J Hand Surg Am 2003;28:464-8.

19. Kadar A, Thoreson AR, Reisdorf RL, et al. Turkey model for flexor tendon research: in vitro comparison of human, canine, turkey, and chicken tendons. J Surg Res 2017;216: 46-55. 